\title{
Características clínicas y paraclínicas de las neoplasias mieloproliferativas crónicas cromosoma Filadelfia negativas
}

\author{
Clinical and paraclinical characteristics of chronic \\ myeloproliferative neoplasms Philadelphia \\ chromosome negative
}

\author{
Julio César Solano, Claudia Patricia Casas, Virginia Abello, \\ María Helena Solano • Bogotá, D.C. (Colombia)
}

\section{Resumen}

Objetivo: describir las características clínicas y paraclínicas de los pacientes con neoplasias mieloproliferativas crónicas cromosoma Filadelfia negativa valorados en la consulta externa de hematología del Hospital de San José desde enero de 2005 hasta mayo de 2010.

Material y métodos: estudio de serie de casos en el que se incluyeron los pacientes diagnosticados con neoplasias mieloproliferativas crónicas cromosoma Filadelfia negativas.

Resultados: un total de 34 pacientes con neoplasias mieloproliferativas (NM) cromosomas Filadelfia negativas fueron identificados. El principal diagnóstico encontrado fue de trombocitemia esencial en 17 pacientes (50\%), policitemia Vera Rubra en seis pacientes (17.6\%), neoplasia mieloproliferativa asociadas a eosinofilia en seis pacientes (17.6\%), mielofibrosis primaria en tres pacientes $(8.8 \%) \mathrm{Y}$ neoplasias mieloproliferativas no clasificables en dos pacientes (5.8\%). La mediana de edad fue de 63.5 años (RIQ: 51 a 74) y 21 pacientes $(61.7 \%)$ correspondían al sexo femenino. Dos pacientes del número total progresaron a mielofibrosis $(5.8 \%)$, ningún paciente desarrolló leucemia aguda. Veintisiete pacientes $(79.4 \%)$ recibieron hidroxiurea como manejo farmacológico principal. Catorce pacientes presentaron complicaciones $(41.1 \%)$, de los cuales cinco fueron episodios trombóticos $(14.7 \%)$, tres episodios hemorrágicos $(8.8 \%)$, tres pacientes presentaron hipertensión pulmonar $(8.8 \%)$ y un paciente desarrolló vértigo (2.9\%). Finalmente el tiempo desde el diagnóstico hasta la aparición de complicaciones fue de 19.55 meses (RIQ: 8-50.23)

Conclusiones: las neoplasias mieloproliferativas crónicas cromosoma Filadelfia negativas son patologías muy raras, el mayor número se agrupan en trombocitemia esencial, policitemia Vera y neoplasias asociadas a eosinofilia. La principal opción terapéutica es la hidroxiurea con una baja toxicidad. No es posible analizar la presencia de las mutaciones tirosina-kinasas (JAK2 V617F, PGDFRA, PDGFRB y FGFR1) ya que son herramientas de reciente ingreso al arsenal diagnóstico y cuyo impacto como factor pronóstico o terapéutico se encuentra en estudio. Las complicaciones más frecuentemente encontradas en esta serie son los eventos trombóticos venosos. (Acta Med Colomb 2012; 37: 66-73)

Palabras clave: desórdenes mieloproliferativos, policitemia Vera, trombocitemia esencial, mielofibrosis primaria, mutación JAK-2V617F, neoplasias mieloproliferativas asociadas con eosinofilia.

\footnotetext{
Abstract

Objective: describe features clinics and patients with chronic myeloproliferative neoplasm chromosome Philadelphia negative valued at the Hospital San Jose hematology outpatient from January 2005 until May 2010.

Materials and methods: Studio series case included patients diagnosed with chronic myeloproliferative neoplasm chromosome Philadelphia negative.

Results: A total of 34 chronic myeloproliferative neoplasm chromosome Philadelphia negative patients were identified. The main found diagnosis was Essential Thrombocythemia in 17 patients (50\%), Polycythemia Rubra Vera in six patients (17.6\%), chronic myeloproliferative neoplasm associ-
}

Dr. Julio César Solano Vega: Residente de Hematología; Dras. Claudia Patricia Casas Patarroyo y Virginia Abello Polo: Instructoras Asistentes, Hematólogas; Dra. María Helena Solano: Instructora Titular. Facultad de Medicina, Fundación Universitaria de Ciencias de la Salud, Hospital de San José. Bogotá, D.C. (Colombia)

Correspondencia. Dr. Julio César Solano Vega. Hospital de San José. Bogotá, D.C. (Colombia)

E-mail: solanovega@gmail.com Recibido: 11/XI/2011 Aceptado: 07/VI/2012 
ated with eosinophilia in six patients (17.6\%), myelofibrosis primary in three patients $(8.8 \%)$ and chronic myeloproliferative neoplasm not classifiable in two patients $(5.8 \%)$. The median age was 63.5 years (R: 51-74) and 21 patients (61.7\%) were female. Two patients in the total number progressed Myelofibrosis (5.8\%), no patient acute leukemia development. Twenty-seven patients (79.4\%) received Hydroxyurea as main pharmacological management. Fourteen patients presented complications $(41.1 \%)$, of which five were thrombotic episodes $(14.7 \%)$, three bleeding episodes $(8.8 \%)$, three patients had pulmonary hypertension $(8.8 \%)$ and one patient developed Vertigo (2.9\%). Finally the time since diagnosis until the occurrence of complications was 19.55 months (R: 8-50.23).

Conclusión: neoplasm Mieloproliferativas Chronicles are very rare pathologies, as many are grouped into essential Thrombocythemia, Polycythemia Vera and neoplasms associated with Eosinophilia. The main therapeutic option is with a low toxicity Hydroxyurea. It is not possible to analyze the presence of mutations tyrosine kinases (JAK2 V617F PGDFRA PDGFRB, FGFR1) because they are tools of recent entry to the diagnostic arsenal and whose impact as a prognostic factor or therapeutic is in studio. Thrombotic venous events are frequently found in these patients. (Acta Med Colomb 2012; 37: 66-73)

Keywords: Disorders myeloproliferative, Polycythemia vera, Essential thrombocythemia, Myelofibrosis primary, Neoplasm associated with Eosinophilia. Mutation JAK-2V617F.

\section{Introducción}

Las neoplasias mieloproliferativas crónicas cromosoma Filadelfia negativas son desórdenes de células madre hematopoyéticas clonales caracterizadas por la proliferación de una o más líneas (granulocítica, eritroide, megacariocítica y mastocitos). Su pico de incidencia se encuentra entre la quinta y séptima décadas de la vida. Esta incidencia de todos los subtipos combinada es de 6-10 x 100.000 personas anualmente en el mundo (1). En Colombia el Instituto Nacional de Cancerología reportó 12 casos de neoplasias mieloproliferativas para el año 2008 (2). En la última clasificación de la OMS de 2008, se cambió el nombre de enfermedades mieloproliferativas por Neoplasias, las cuales se caracterizan por hipercelularidad de la médula ósea con efectiva maduración hematopoyética e incremento en el número de granulocitos, eritrocitos y/o plaquetas en la sangre periférica. La esplenomegalia y la hepatomegalia son características comunes de este tipo de patologías, causadas por el secuestro de las células sanguíneas o proliferación de células hematopoyéticas anormales (3).

La mayoría si no todas estas neoplasias están asociadas con anormalidades clonales que involucran genes de citoplasma o receptores de fosfotirosina kinasas (PTK).

Las proteínas de la familia JAK son tirosina-kinasas citoplasmáticas, reciben el nombre de Janus (dios romano de dos caras), ya que tiene dos dominios (uno de activación kinasa y otro de inactivación catalítica pseudokinasa). Hasta la fecha se han identificado cuatro JAKs: JAK1, JAK2, JAK3 y TYK2. Estos receptores de citokina tipo I tienen escasa actividad tirosina kinasa intrínseca, pero cuando un ligando se une al receptor, un cambio conformacional atrae varías proteínas JAK2 cercanas y juntas permiten que cada una active a la otra. La JAK2 fosforilada actúa como una PTK activada y comienza a estimular los sitios de carga de las proteínas STAT. Estas proteínas activadas forman dímeros y entran al núcleo, dónde actúan como factores de transcripción para regular genes blanco. El sistema JAK2STAT es el paso crucial en la señalización de numerosas citokinas, incluyendo eritropoyetina, hormona de crecimiento, prolactina, trombopoyetina, factor estimulante de colonia granulocito-macrófago, IL-3 e IL-5 (4).

En 2005 se realiza el descubrimiento de la mutación de la tirosina-kinasa JAK2 V617F, considerada como la mutación constitutiva fundamental que relaciona las tres neoplasias mieloproliferativas crónicas principales: policitemia Vera rubra (PV), trombocitemia esencial (TE) y mielofibrosis primaria (MFP). Esta mutación clonal recurrente en el dominio pseudokinasa conduce a una sustitución de valina por fenilalanina en la posición 617 (JAK2 V617F), lo que produce una fosforilación constitutiva del JAK2 y Ba/F3 o $\mathrm{Ba} / \mathrm{F} 3-E p o R$ permitiendo que varias líneas celulares sobrevivan y proliferen independiente de las citokinas (5). Desde su detección inicial, la mutación JAK2 V617F ha sido descrita en más de $95 \%$ de pacientes con policitemia Vera y en 50$60 \%$ de pacientes con trombocitemia esencial y mielofibrosis primaria. La mutación ocurre en una célula madre común a una célula madre hematopoyética y célula endotelial denominada hemangioblasto. Especialmente a niveles de células madre hematopoyéticas y progenitoras, la hematopoyesis de pacientes incluye clones tipo salvaje, heterocigotos JAK2 V617F y eventualmente homocigotos (6). Algunos autores dudan que el JAK2 V617F sea el evento inicial en estas neoplasias, sin embargo, los resultados de modelos murinos y la alta prevalencia de la mutación en PV, TE y MFP son fuertes argumentos en favorecer esta teoría (4).

¿Cómo una mutación puede originar varias enfermedades? En la mayoría de pacientes con policitemia Vera, el JAK2 V617F es bialélico como consecuencia de una recombinación mitótica mientras que la mutación monoalélica está en la mayoría de pacientes con trombocitemia esencial.

Hay una proporción de pacientes con estos desórdenes que son negativos para la mutación cuando las pruebas 
para alelos específicos son usadas. Diversos investigadores tienen la hipótesis que estos pacientes resultan de mutaciones somáticas que activan la señalización de la tirosina kinasas JAK2 en una forma análoga a la kinasa JAK2617F. La expresión de mutaciones del exón JAK2 ha sido reportada, incluida mutaciones de pérdida de la sensibilidad, deleciones e inserciones involucrando residuos del 538 al 543 (7).

Hay otras tres entidades poco frecuentes que comparten la característica de la formación de un gen de fusión que sintetiza una tirosina kinasa aberrante llamadas neoplasias mieloproliferativas con eosinofilia que encierran anormalidades del PDGFRA, PDGFRB o FGFR1. La eosinofilia es característica pero no invariable y se ha establecido que en el caso de las neoplasias del PDGFRA o FGFR1 la célula de origen es una célula madre pluripotente (linfoide o mieloide) mutada, no está claro si esto sucede con la neoplasia PDGFRB. La neoplasia mieloproliferativa asociada a eosinofilia más común es la que presenta el rearreglo del FIP1L1-PDGFRA formada como resultado de una deleción críptica en el brazo largo del cromosoma $4 q 12(3)$.

Debido a que en Colombia no hay información de estas patologías, es necesario realizar una radiografía de los pacientes del Hospital de San José de Bogotá, el cual es un centro de referencia de hematología en la ciudad, como primer paso para su caracterización y con ello, plantear estrategias de estudio y tratamiento.

\section{Material y métodos}

Los pacientes elegibles se identificaron por medio de la revisión manual del archivo del servicio de hematología del Hospital de San José. Se define neoplasia mieloproliferativa como la proliferación clonal de células madre hematopoyéticas en una o más líneas celulares (leucocitosis, trombocitosis o eritrocitosis), las cuales producen una diversidad de síntomas y que culminan en su gran mayoría con fibrosis de la médula ósea o progresión a leucemia aguda y falla medular secundaria. Se incluyeron los pacientes mayores de 18 años con los siguientes tipos de neoplasias mieloproliferativas: policitemia Vera, trombocitemia esencial, mielofibrosis idiopática, leucemia eosinofílica crónica, neoplasia mieloproliferativa asociadas a eosinofilia (con rearreglo PDGFRA, PDGFRB y FGFR1) y neoplasias mieloproliferativas no clasificables. Las características del cuadro hemático en el momento del diagnóstico y luego de seis meses de tratamiento, los hallazgos de médula ósea, la presencia de esplenomegalia, la presencia de mutación del JAK2, los tipos de tratamiento, duración, suspensión y las complicaciones fueron registradas. Se emplearon medidas de tendencia central y dispersión para las variables numéricas; para las variables cualitativas se utilizaron porcentajes. Los procedimientos que se describen a continuación se tabularon en Microsoft Excel ${ }^{\circledR}$ y se analizaron en el paquete estadístico Stata ${ }^{\circledR}$ versión 10.0. El estudio fue aprobado por el comité de investigaciones de la Facultad de Medicina de la Fundación Universitaria de Ciencias de la Salud.

\section{Resultados}

Un total de 34 pacientes con neoplasias mieloproliferativas crónicas cromosomas Filadelfia negativas fueron identificados desde el $1^{\text {o }}$. de enero de 2005 hasta el 31 de mayo de 2010. Los criterios de clasificación para neoplasias mieloproliferativas de la OMS fueron cumplidos en el $91.18 \%$. La mediana de edad fue de 63.5 años (RIQ: 51 a 74) y el $55.88 \%$ fueron mayores de 61 años. Del total de pacientes, 21 pacientes $(61.7 \%)$ correspondían al sexo femenino. El principal diagnóstico encontrado en esta serie de pacientes fue de trombocitemia esencial en 17 pacientes (50\%), en segundo término se encontraron con un número similar de pacientes (seis pacientes cada una), policitemia Vera rubra y neoplasia mieloproliferativa asociadas a eosinofilia. Mielofibrosis fue el principal diagnóstico en tres pacientes $(8.8 \%)$ y neoplasias mieloproliferativas no clasificables en dos pacientes (5.8\%). No se encontró casos de leucemia eosinofílica crónica, ni neoplasias asociadas a las mutaciones PDGFRA, PDGFRB y FGFR1 (Tabla 1). Dos pacientes progresaron a mielofibrosis $(5.8 \%)$, mientras que ningún paciente progresó a leucemia aguda.

De los pacientes que presentaron trombocitemia esencial, 12 pacientes $(70.5 \%)$ eran mujeres. La mediana de plaquetas en el momento del diagnóstico fue de $1190 \mathrm{x}$ 109/l (RIQ: $\left.1052 \times 10^{9} / 1-1423 \times 10^{9} / 1\right)$. Ninguno de estos pacientes presentaba esplenomegalia. La prueba para la mutación JAK 2 V617F fue realizada en siete pacientes y sus resultados fueron negativos. El $78.5 \%$ de estos pacientes tenía como hallazgo una médula ósea hipercelular para la edad, con una mediana de 70\% (RIQ: 60\% a 80\%), asociado a esto $92.86 \%$ (13 pacientes de 15 con datos de biopsia de médula ósea) presentaba hiperplasia de la línea megacariocítica. Todos los pacientes con trombocitemia esencial recibieron manejo farmacológico (Tabla 2). Quince pacientes $(88.2 \%)$ recibieron hidroxiurea, con una mediana de duración de 24 meses (RIQ: 9-48), a una dosis de $1000 \mathrm{mg}$ al día (RIQ: 500-1500) y sólo uno la suspendió por sangrado digestivo alto. Doce pacientes (70.5\%) recibieron ASA, con una duración de dicha terapia de 24 meses (RIQ: 18-30.5). A los seis meses de tratamiento, la mediana de plaquetas era de $684 \times 10^{9} / 1$ (RIQ: $\left.506 \times 10^{9} / 1-758 \times 10^{9}\right)$ con una reducción absoluta en el recuento plaquetario de $448 \times 10^{9} / 1$ (RIQ: $152 \times 10^{9}-660$ x $\left.10^{9} / 1\right)$. El tiempo desde el diagnóstico hasta la presencia de complicaciones fue de 19.5 meses (RIQ:17.1-34.1), se presentaron dos complicaciones hemorrágicas (hematoma en muslo y hemorragia de vías digestivas altas) y una complicación trombótica (tromboembolismo pulmonar), además de un episodio de vértigo.

De seis pacientes con policitemia Vera Rubra se encontraron cuatro mujeres $(66.6 \%)$, con una mediana de edad 
Tabla 1. Características demográficas y clínicas.

\begin{tabular}{|lc|}
\hline & $\mathbf{n}=\mathbf{3 4}$ \\
\hline Sexo & \\
$\quad$ Masculino & $13(38.24)$ \\
Femenino & $21(61.76)$ \\
\hline Edad (años)* & $63.5(51-74)$ \\
$\quad$ Grupos de edad & \\
$\quad$ Menores o iguales a 40 años de edad & $2(5.8)$ \\
$\quad 41$ a 60 años de edad & $13(38.2)$ \\
$\quad$ Mayores de 61 años de edad & $19(55.8)$ \\
\hline Tipo de neoplasia mieloproliferativa & $6(17.6)$ \\
Policitemia Vera Rubra & $17(50)$ \\
Trombocitemia esencial & $3(8.8)$ \\
Mielofibrosis primaria & $6(17.6)$ \\
\hline Neoplasia mieloproliferativa asociado a eosinofilia & $2(5.8)$ \\
\hline Neoplasia mieloproliferativa no clasificable & \\
\hline Cumple criterios 2002-2008 OMS & $31(91.1)$ \\
\hline Si & $2(5.8)$ \\
\hline Progresión a mielofibrosis, $\mathbf{~ ( \% ) ~}$ & $19.5(8-50.2)$ \\
Si & \\
\hline Tiempo desde diagnóstico hasta complicación en meses * & \\
\hline * Mediana (RI = rango intercuartílico) & \\
\hline
\end{tabular}

72 años (RIQ: 67-75). Cinco pacientes no se les realizó la mutación para el JAK2 V617F y la mitad de pacientes tenían esplenomegalia al examen físico. La mediana de hemoglobina a los seis meses al momento del diagnóstico fue de $16.5 \mathrm{~g} / \mathrm{dL}$ (RIQ: 16.4-17-3), con una reducción absoluta de $1.9 \mathrm{~g} / \mathrm{dL}$ (RIQ: 0.4-3-4). Cuatro pacientes presentaban una médula ósea hipercelular, con una mediana de $80 \%$ (RIQ: $70 \%-100 \%$ ), cinco pacientes tenían hiperplasia megacariocítica y tres presentaban hiperplasia eritroide, mientras que sólo dos presentaban hiperplasia granulocítica. Los seis pacientes recibieron tratamiento, descritos en la Tabla 3. Cinco recibieron flebotomías, a una dosis de $250 \mathrm{~mL}$ cada quince días, con una duración de 12 meses (RIQ: 12-48). Todos requirieron hidroxiurea, a dosis de $1000 \mathrm{mg}$ al día y una mediana de duración de 60 meses (RIQ: 12-120). Finalmente, un solo paciente recibió interferón alfa 2 a pegilado por espacio de seis meses. Las complicaciones se presentaron posterior al diagnóstico en un tiempo con una mediana de 2.2 meses (RIQ: 0.16-4.5) luego del diagnóstico, principalmente de origen trombótico.

Seis pacientes tenían diagnóstico de neoplasia mieloproliferativa asociada a eosinofilia. La mediana de edad fue de 51 años (RIQ: 48-65), el 50\% eran mujeres y a un solo paciente se realizó la mutación del PDGFRA la cual fue negativa. Los eosinófilos en sangre periférica al momento del diagnóstico fueron de 5.18 x $10^{9}$ (RIQ: 1.9 x 109/1-9.0 x $\left.10^{9} / 1\right)$. Ninguno presentó esplenomegalia. Cuatro pacientes tenían como hallazgo una médula hipercelular, con una mediana de $80 \%$ (RIQ: $65 \%-95 \%$ ), dos presentaron blastos y la mediana de eosinófilos era de $16.1 \%$ (RIQ: $13 \%-27.9 \%$ ). Cinco pacientes $(83.3 \%)$ recibieron tratamiento farmaco-
Tabla 2. Trombocitemia esencial.

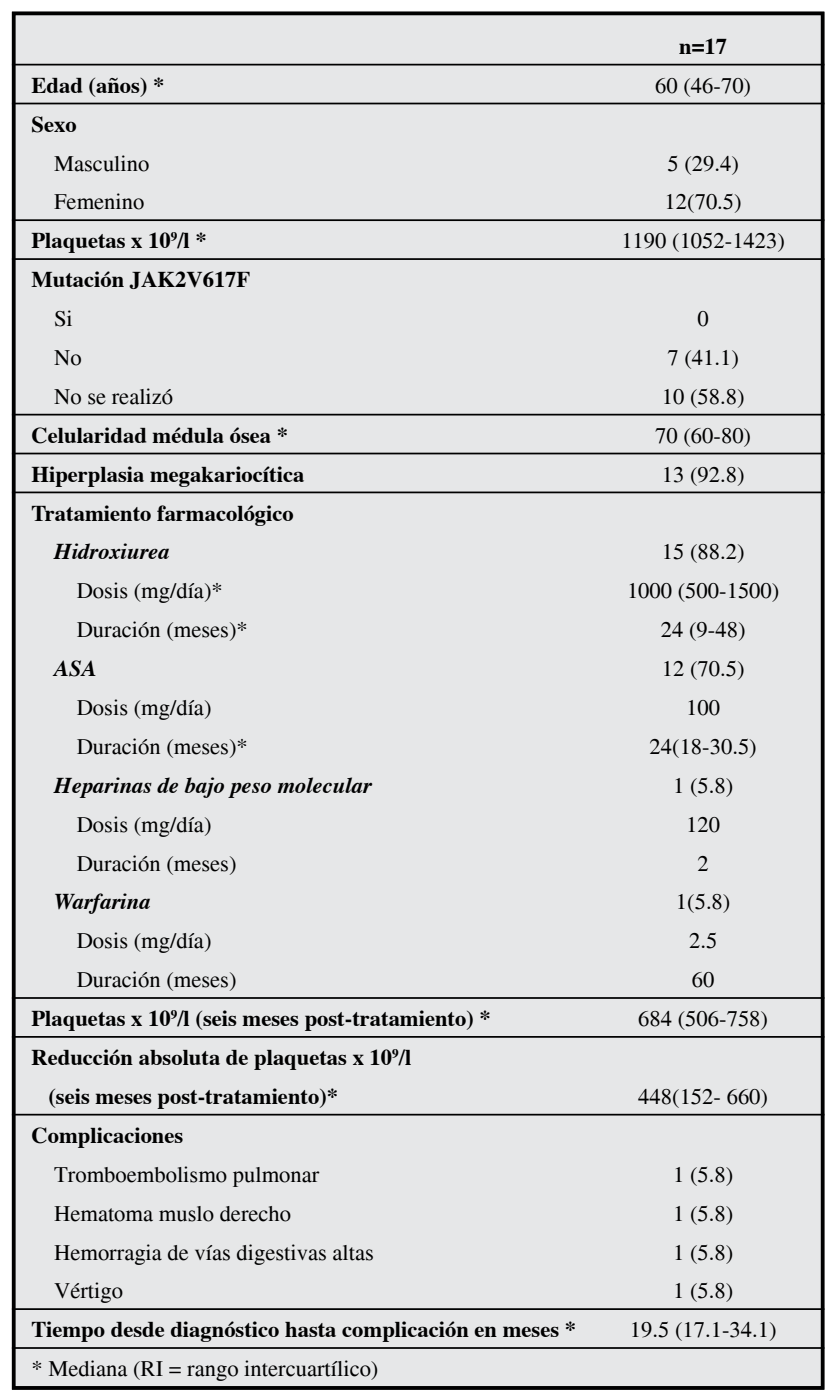

lógico (Tabla 4). Cuatro pacientes recibieron hidroxiurea a dosis de $1000 \mathrm{mg}$ /día (RIQ: 750-1000) por espacio de 27.5 meses. La mitad de pacientes fueron manejados con prednisolona, a dosis mediana de $25 \mathrm{mg}$ (RIQ: 15-55). La reducción absoluta de eósinofilos fue de $2.6 \times 10^{9}$ (Rango: $0.3 \times 10^{9}-4.9 \times 10^{9}$ ) con una mediana a los seis meses postratamiento de $3.3 \times 10^{9}$ (Rango: $0.8 \times 10^{9}-4.0 \times 10^{9}$ ). Las complicaciones fueron principalmente trombóticas (Tabla 4).

Sólo tres pacientes tenían diagnóstico de mielofibrosis primaria, dos eran hombres. La mediana de hemoglobina al diagnóstico fue de $11.5 \mathrm{~g} / \mathrm{dL}(8.1 \mathrm{~g} / \mathrm{dL}-13.4 \mathrm{~g} / \mathrm{dL})$. Todos tenían esplenomegalia, con un diámetro promedio de 24 $\mathrm{cm}$. Un solo paciente tenía leucoeritroblastos en sangre periférica, así mismo un solo paciente tenía una médula ósea hipercelular e hiperplasia megacariocítica. Todos los pacientes recibieron tratamiento, dos recibieron eritropoyetina a dosis de 12000 UI semanales, otro recibió hidroxiurea, ASA y talidomida. La hemoglobina seis meses 
Tabla 3. Policitemia Vera.

\begin{tabular}{|c|c|}
\hline & $\mathrm{n}=6$ \\
\hline \multicolumn{2}{|l|}{ Sexo } \\
\hline Masculino & $2(33.3)$ \\
\hline Femenino & $4(66.6)$ \\
\hline Edad (años)* & $72(67-75)$ \\
\hline $\mathrm{Hb}(\mathrm{g} / \mathrm{dL})^{*}$ & $19.05(18.1-19.9)$ \\
\hline \multicolumn{2}{|l|}{ Mutaciòn JAK2V617F } \\
\hline $\mathrm{Si}$ & 0 \\
\hline No & $1(16.6)$ \\
\hline No se realizó & $5(83.3)$ \\
\hline Celularidad médula osea & $80(70-100)$ \\
\hline \multicolumn{2}{|l|}{ Tratamiento } \\
\hline Flebotomia & $5(83.3)$ \\
\hline Dosis (cc/día) & 500 \\
\hline Duración (meses) * & $12(12-48)$ \\
\hline Hidroxiurea & $6(100)$ \\
\hline Dosis (mg/día) & $1000(500-1000)$ \\
\hline Duración meses & $60(12-120)$ \\
\hline$A S A$ & $4(66.6)$ \\
\hline Dosis (mg/día) & 100 \\
\hline Duración meses * & $36(12-60)$ \\
\hline Warfarina & $1(16.67)$ \\
\hline Dosis (mg/día) & 2.5 \\
\hline Duración meses & 36 \\
\hline Interferon alfa 2 a pegilado & $1(16.6)$ \\
\hline Dosis (mcg/semanal) & 180 \\
\hline Duración de interferón (meses) & 6 \\
\hline Hb $(g / d L)$ seis meses post-tto * & $16.5(16.4-17.3)$ \\
\hline Reducción de $\mathrm{Hb}(\mathrm{g} / \mathrm{dL})$ seis meses post-tto & $1.9(.4-3.4)$ \\
\hline \multicolumn{2}{|l|}{ Complicaciones } \\
\hline Tromboembolismo pulmonar & $1(16.6)$ \\
\hline Hipertensión pulmonar leve & $1(16.6)$ \\
\hline Hipertensión portal & $1(16.6)$ \\
\hline Ataque cerebro-vascular isquémico & $1(16.6)$ \\
\hline Tiempo desde diagnóstico hasta complicación en meses* & $2.2(0.16-4.5)$ \\
\hline * Mediana (RI = rango intercuartílico) & \\
\hline
\end{tabular}

post-tratamiento fue de $8.3 \mathrm{~g} / \mathrm{dL}$ (RIQ: 7.5-11.9), con una reducción absoluta de $0.2 \mathrm{~g} / \mathrm{dL}$, con una sola complicación (hipertensión pulmonar severa) a los 51.7 meses de realizado el diagnóstico.

Se encontraron dos pacientes con diagnóstico de neoplasia mieloproliferativa no clasificable de sexo femenino, a ninguna se realizó la mutación JAK2 V617. Una de las pacientes tenía esplenomegalia, la médula ósea de las dos pacientes era hipercelular, una con hiperplasia megacariocítica y la otra paciente presentaba hiperplasia granulocítica. La reducción de leucocitos con el tratamiento fue de $0.2 \times 10^{9} / 1$ y $11.7 \times 10^{9} / 1$ en cada paciente (Tabla 6).

\section{Discusión}

En nuestro medio es usual extrapolar los resultados de series extranjeras al manejo de los pacientes; este trabajo es uno de los primeros acercamientos a las neoplasias mieloproliferativas crónicas cromosoma Filadelfia negativas
Tabla 4. Neoplasias mieloproliferativas/síndrome hipereosinofilico.

\begin{tabular}{|c|c|}
\hline & $\mathrm{n}=6$ \\
\hline Edad (años) mediana & $51(48-65)$ \\
\hline \multicolumn{2}{|l|}{ Sexo } \\
\hline Masculino & $3(50)$ \\
\hline Femenino & $3(50)$ \\
\hline Eosinofilos al momento del diagnóstico * & $5181(1900-9000)$ \\
\hline Celularidad de la médula ósea * & $80(65-95)$ \\
\hline Eósinofilos en médula ósea * & $16.1(13-27.9)$ \\
\hline \multicolumn{2}{|l|}{ Tratamiento farmacológico } \\
\hline Hidroxiurea & $4(80)$ \\
\hline Dosis (mg/día)* & $1000(750-1000)$ \\
\hline Duración hidroxiurea (meses)* & $27.5(13.5-36)$ \\
\hline Prednisolona & $3(60)$ \\
\hline Dosis (mg/día)* & $25(15-55)$ \\
\hline Duración (meses)* & $19(12-36)$ \\
\hline Warfarina & $2(40)$ \\
\hline Dosis (mg/día)* & $6.2(5-7.5)$ \\
\hline Duración (meses)* & $10(8-12)$ \\
\hline Eósinofilos $\times 10^{9} / \mathrm{l}$ seis meses post -tto * & $3345(861-4000)$ \\
\hline Reducción absoluta de eosinófilos x $10^{9} / 1$ seis meses post-tto * & $2645(303-4900)$ \\
\hline \multicolumn{2}{|l|}{ Complicaciones } \\
\hline Trombosis venosa profunda & $1(25)$ \\
\hline Asma & $1(25)$ \\
\hline Hipertensión pulmonar severa & $1(25)$ \\
\hline Trombo intracavitario ventrículo izquierdo & $1(25)$ \\
\hline Tiempo desde diagnóstico hasta complicación en meses * & 34.06 \\
\hline * Mediana $(\mathrm{RI}=$ rango intercuartílico $)$ & \\
\hline
\end{tabular}

Tabla 5. Mielofibrosis primaria.

\begin{tabular}{|lc|}
\hline & $\mathrm{n}=3$ \\
\hline Edad (años) mediana & $75(59-81)$ \\
\hline Sexo & \\
Masculino & $2(66.6)$ \\
Femenino & $1(33.3)$ \\
\hline Hb (g/dL) al momento del diagnóstico * & $11.5(8.1-13.4)$ \\
\hline Mutación JAK2V617F & \\
Sí & 0 \\
No & $2(66.6)$ \\
No se realizó & $1(33.33)$ \\
\hline Tratamiento farmacológico & \\
Eritropoyetina & $2(66.6)$ \\
Dosis (UI/semanal) & 12000 \\
Duración (meses) & $18(12-24)$ \\
Hidroxiurea & $1(33.3)$ \\
Dosis (mg/día) & 1500 \\
Duración (meses) & 6 \\
ASA & $1(33.3)$ \\
Dosis (mg/día) & 100 \\
Duración (meses) & 24 \\
Talidomida & $1(33.3)$ \\
Dosis (mg/día) & 50 \\
Duración (meses) & 12 \\
\hline Hb (g/dL) seis meses post-tratamiento * & $5.3(7.5-11.9)$ \\
\hline Descenso de Hb (g/dL) seis meses post-tratamiento * & \\
\hline Tiempo desde diagnóstico hasta complicación en meses * & \\
\hline Mediana (RI = rango intercuartílico) & \\
\hline
\end{tabular}


Tabla 6. Neoplasia mieloproliferativa no clasificable.

\begin{tabular}{|c|c|}
\hline & $\mathrm{n}=\mathbf{2}$ \\
\hline Edad (años) * & $67(58-76)$ \\
\hline \multicolumn{2}{|l|}{ Sexo } \\
\hline Masculino & 0 \\
\hline Femenino & $2(100)$ \\
\hline Leucocitos $\times 10^{9} / \mathrm{L}$ al diagnóstico * & $31.4(15.7-47.2)$ \\
\hline Celularidad de médula ósea * & $85(70-100)$ \\
\hline \multicolumn{2}{|l|}{ Tratamiento farmacológico } \\
\hline Hidroxiurea & $1(50)$ \\
\hline Dosis (mg/día) & 500 \\
\hline Duración (meses) & 6 \\
\hline Eritropoyetina & $1(50)$ \\
\hline Dosis (UI/semanal) & 12000 \\
\hline Duración de eritropoyetina (meses) & 6 \\
\hline Leucocitos x $10^{9} / /$ seis meses post-tto mediana * & 25.5(15.5-35.5) \\
\hline Reducción de leucocitos x $10^{9}$ seis meses post-tto & $5.9(0.2-11.7)$ \\
\hline \multicolumn{2}{|l|}{ Complicaciones } \\
\hline HVDA & $1(50)$ \\
\hline Hipertensión portal & $1(50)$ \\
\hline Tiempo desde diagnóstico hasta complicación en meses * & $5.3(4.56-6.06)$ \\
\hline * Mediana $(\mathrm{RI}=$ rango intercuartílico) & \\
\hline
\end{tabular}

en Colombia. El estudio se desarrolló en un solo centro hospitalario de referencia, con un tiempo de evaluación de cinco años y por ser patologías de baja frecuencia, se evaluaron en conjunto y no de manera separada como lo reporta la literatura mundial.

La mitad de los 34 pacientes encontrados en nuestro trabajo fueron diagnosticados con trombocitemia esencial, nueve pacientes eran de alto riesgo, los cuales requerían de terapia para reducción del recuento plaquetario; sólo uno no recibió citorreducción. Siete de los ocho pacientes sin factores de riesgo que no requerían de inicio de tratamiento para reducción del recuento plaquetario, recibieron hidroxiurea. Aunque para el manejo de estos pacientes el Consenso de la Sociedad de Hematología Italiana (8) hace una serie de recomendaciones para definir el inicio de manejo farmacológico, es clara la preocupación de los médicos tratantes frente a recuentos plaquetarios mayores a un millón, lo que hace que estos pacientes terminen recibiendo tratamiento.

En dos estudios de cohortes con pacientes de alto riesgo, la hidroxiurea demostró su eficacia antitrombotica, encontrando en el grupo de hidroxiurea complicaciones alrededor de $1.5 \%$ /paciente-año comparada con el grupo no tratado de $7.4 \%$ /paciente-año $(9 ; 10)$. Es claro el beneficio de este fármaco, a este respecto la guía antes mencionada, recomienda a pacientes mayores de 60 años, con factores de riesgo cardiovascular, trombofilia familiar o recuento plaquetario excesivamente alto (plaquetas $>1500 \times 10^{9} / 1$ ) administrar hidroxiurea como primera opción terapéutica (recomendación grado A). Mientras que para pacientes jóvenes, menores de 60 años que requieran terapia el uso de anagrelide o interferón alfa 2 a pegilado, surge como opción ante el potencial riesgo de transformación a malignidad de la hidroxiurea (Recomendación D) (8) . Esta última recomendación es debatida y faltan estudios que soporten esta opción terapéutica.

En nuestro estudio se logró una mediana de reducción en el recuento plaquetario con el uso de hidroxiurea de 684 x $10^{9}$ (RIQ: 506-758). No hay una evidencia fuerte que recomiende un recuento específico que vaya a proteger al paciente de una complicación, no obstante por el cambio en los criterios de la OMS de 2008 , de 450 x $10^{9} / 1$ a 600 x $10^{9} / 1$ es una meta globalmente recomendada; luego de seis meses de terapia se logró una reducción cercana a estos valores.

De los 12 pacientes que recibieron ASA (70.59\%), sólo cuatro se clasificaron como alto riesgo. Para la terapia de antiagregación sólo hay datos retrospectivos (11) y de éstos se deriva una recomendación (grado B) en pacientes con síntomas microcirculatorios, evento vascular reciente arterial, evidencia de enfermedad arterial coronaria reciente o en trombocitosis extrema (plaquetas $>1500 \times 10^{9} / 1$ ), los cuales deben recibir antiagregación con ASA y concomitantemente terapia citorreductora (8). En pacientes de bajo riesgo no parece haber ningún beneficio. La necesidad de que los pacientes reciban esta terapia recae en la conducta clínica habitual de evitar fenómenos trombóticos, en esta serie de casos parece por el contrario que estamos dejando sin antiagregación a un número importante de pacientes; sin embargo, el criterio médico y la individualización son siempre importante en patologías tan infrecuentes como éstas.

En relación con las neoplasias mieloproliferativas asociadas a eosinofilia no hay evidencia de su incidencia en el mundo. Los pacientes que se presentan con signos y síntomas de síndrome hipereosinofílico pueden caer en una de cuatro clasificaciones propuestas por la OMS: neoplasias mieloproliferativas/SHE, sindrome mieloproliferativo/leucemia eosinofílica crónica, neoplasias mieloides asociadas con eosinofilia y anormalidades del PDGFRA, PDGFRB o FGFR1 y neoplasia/linfoma T no clasificable. Esta clasificación tiene utilidad cuando hay un proceso neoplásico clonal bien definido, pero en la mayoría de casos resultan idiopáticas, con las pruebas moleculares negativas como ocurrió en nuestro estudio (12). Encontramos seis pacientes de 34 con esta sospecha diagnóstica, sólo a uno se le realizó la mutación del FIP1L1/ PDGFRA la cual fue negativa. En cuatro casos se administró hidroxiurea y tres prednisolona. Los algoritmos actuales de cuándo iniciar terapia no son claros, expertos como el Dr. Tefferi recomiendan no iniciar tratamiento a menos que haya compromiso de órgano blanco (en esta serie de casos no se reporta por la escasez de datos en la historia clínica) o recuentos tan altos como eosinófilos mayores de 30 x 10\% 1 y además indica que para el manejo de eosinofilia idiopática el medicamento de elección es prednisolona usando como ahorrador hidroxiurea; cuando no se lograr estabilizar el 
cuadro con dosis menores a $10 \mathrm{mg} /$ día. Otras opciones por contemplar son el interferon alfa pegilado, alemtuzumab o mepolizumab. En caso de eosinofilia clonal recomienda el uso de imatinib (13). No hay evidencia que soporte cual es el recuento de seguridad como objetivo de la terapia, de nuevo, en relación con los criterios de clasificación de la OMS, recuentos menores de $1.5 \times 10^{9} / \mathrm{L}$ parecen seguros. Los resultados de eosinófilos luego de seis meses de tratamiento en esta serie de casos fueron de 3345 (RIQ: 861-4000), no logrando los niveles esperados pero si con adecuado control de síntomas.

En cuanto a la policitemia Vera Rubra, el estudio mostró una mayor incidencia en mujeres que en hombres (cuatro de seis pacientes) en contravía de varios estudios en los cuales los hombres presentan más frecuentemente este subtipo (14). Dicho hallazgo probablemente en relación con el número pequeño de pacientes. Un solo paciente se transformó a mielofibrosis, las diversas series muestran un número mayor de pacientes que progresan a leucemia aguda (15), no encontrado en nuestro trabajo, en relación probablemente al poco tiempo de observación. Todos nuestros pacientes con PV recibieron manejo con hidroxiurea; suspendido en uno por presencia de trombocitopenia secundaria a mielofibrosis, el cual requirió el uso de interferón alfa 2 a pegilado. Los estudios con esta molécula muestran respuestas bastante pobres con empeoramiento de las citopenias (16). En nuestro paciente hubo estabilidad de la hemoglobina, pero sin empeoramiento de la trombocitopenia, finalmente se suspendió a los seis meses. La flebotomía fue utilizada en cinco pacientes, con dosis altas cada dos semanas. El impacto de estas dos terapias (flebotomía e hidroxiurea) produjo a los seis meses normalización de los niveles de hemoglobina $16.55 \mathrm{~g} / \mathrm{dL}$ (Rango: 16.4-17.3). El ECLAP (European Collaborative for Low Dose Aspirin in Polycythemia Vera) aleatorizó a 518 pacientes a ASA $100 \mathrm{mg} /$ día o placebo, mostrando reducción en la incidencia de ACV, IAM, embolia pulmonar $y$ trombosis venosa profunda en cinco años (17). En nuestro estudio cuatro pacientes recibieron ASA a la dosis usual y encontramos complicaciones tromboembólicas venosas en dos pacientes (ACV y TEP). Hay que mencionar la presencia de hipertensión pulmonar y portal en dos pacientes, no es claro actualmente el manejo o si la terapia citorreductora mejora este fenómeno (18).

Tres pacientes con el diagnóstico de mielofibrosis primaria se presentaron en esta serie de casos, los cuales recibieron eritropoyetina (dos pacientes), hidroxiurea y talidomida. En diversos estudios se muestra que la talidomida produce disminución del soporte transfusional (19), nuestro paciente tuvo una mejoría a los seis meses de tratamiento de $0.4 \mathrm{~g} /$ $\mathrm{dL}$, sin requerimiento de soporte transfusional. Respecto al uso de la eritropoyetina, un paciente que la recibió no obtuvo mejoría luego de seis meses con un descenso de la hemoglobina de $13.4 \mathrm{~g} / \mathrm{dL}$ a $7.5 \mathrm{~g} / \mathrm{dl}$, el otro paciente tuvo un aumento de $8.1 \mathrm{~g} / \mathrm{dL}$ a $8.3 \mathrm{~g} / \mathrm{L}$. Como lo sugiere la experiencia de 20 años de la Mayo Clinic, la eritropoyetina debería ser evitada en pacientes con hemoglobina mayor de $10 \mathrm{~g} / \mathrm{dL}$, dependientes de soporte transfusional, paciente con niveles de eritropoyetina sérica mayores a $125 \mathrm{U} / \mathrm{L}$, esplenomegalia masiva y ser homocitogotos para la mutación JAK2 V617F (20).

Cinco de los 34 pacientes (14.7\%) presentaron complicaciones trombóticas. Hay una amplia variación en la probabilidad de trombosis mayor en trombocitemia esencial (7.6-29.4\%). Y en policitemia Vera de (11.2-38.6\%) para pacientes con diagnóstico de novo (21). Además en estos pacientes, los eventos arteriales son más comunes que los venosos. En nuestra serie de casos sólo se presentó un evento arterial (ataque cerebrovascular isquémico) y el resto fueron venosos. Tres de los 34 pacientes $(8.82 \%)$ presentaron complicaciones hemorrágicas (dos con diagnóstico de hemorragia de vías digestivas altas y una con hematoma muscular), dos de estos pacientes tenían como diagnóstico trombocitemia esencial. La incidencia de sangrado como manifestación inicial varía entre 3-18\% en trombocitemia esencial y $3-8.1 \%$ en policitemia Vera. Algunas series muestran que las complicaciones hemorrágicas son más frecuentes en pacientes con plaquetas elevadas y uso asociado de ASA (el paciente con el hematoma del muslo tenía $910 \times 10^{9} / 1$ y el paciente con el sangrado digestivo 1477 x $10^{9} / 1$ y uso de ASA). El riesgo alto de sangrado está dado por los siguientes factores: alto riesgo (plaquetas $>1500 \mathrm{x}$ $10 \%$, historia mayor de sangrado o tres factores de riesgo menor), riesgo intermedio (dos factores de riesgo menor) y riesgo menor ( $<1000 \times 10 \%$, duración de la enfermedad de $>15$ años y antecedente de sangrado menor). Según el riesgo los pacientes deben recibir terapia citorreductora y evitar el uso de ASA (22).

La presencia de la mutación JAK2 V617F es una herramienta en el proceso diagnóstico y es criterio mayor de policitemia Vera, trombocitemia esencial y mielofibrosis, en este punto sería de utilidad evaluar la carga de los alelos y correlacionarlo con el fenotipo clínico, pero es validado mundialmente la realización del estudio de esta mutación con una reacción en cadena de la polimerasa (PCR en tiempo real), cualitativa en neutrófilos aislados. Dicha prueba se realizo en siete de nuestros pacientes todos con resultados negativos, aunque no disponemos de técnicas adicionales se propone en el futuro evaluar otro tipo de mutaciones del exón 12 del JAK en pacientes V617F negativos, que en diversos estudios se han encontrado hasta en un $4 \%$ de este tipo de neoplasias (23). La aparición de moléculas tipo inhibidor del JAK como ruxolitinib (actualmente en fase I y II) (24), abren una esperanza a pacientes que no toleran o no responden a la hidroxiurea e interferón pegilado.

Aunque es un número reducido de pacientes, este estudio es un primer acercamiento a las neoplasias mieloproliferativas crónicas cromosoma Filadelfia negativas en Colombia y los datos aquí mostrados permitirán en el futuro diseñar programas tanto para el seguimiento como para la aproximación farmacológica de este tipo de patología. 


\section{Declaración de fuentes de financiamiento y conflictos de interés}

La fuente de financiación de la investigación fue a través de la presupuesto de la Fundación Universitaria de Ciencias de la Salud.

\section{Declaración de conflicto de interés}

Virginia Abello y María Helena Solano: Asesoras externas Novartis. Investigadoras: Estudio TOPS, ENACT.

\section{Referencias}

1. Johansson P. Epidemiology of the myeloproliferative disorders polycithemia vera and essential thrombocythemia. Semin Thromb Hemost 2006; 32: 171-3.

2. Instituto Nacional de Cancerología. Anuario Estadístico 2008-2009. Report No. 6.

3. Swerdlow SH, Campo E, Harris NL, Jaffe ES, Pileri SA, Stein H, Thiele J, Vardiman JW. WHO Classification of Tumors of Haematopoietic and Lymphoid Tissues 2008; WHO IARC: 2008.

4. Vainchenker W, Dusa A, Constantinescu SN. JAKs in pathology: role of Janus kinases in hematopoietic malignancies and immunodeficiencies. Semin Cell Dev Biol 2008; 19(4): 385-93.

5. Smith CA, Fan G. The saga of JAK2 mutations and translocations in hematologic disorders: pathogenesis, diagnostic and therapeutic prospects, and revised World Health Organization diagnostic criteria for myeloproliferative neoplasms. Hum Pathol 2008; 39(6): 795-810.

6. Campbell PJ, Green AR. The myeloproliferative disorders. N Engl J Med 2006; 355(23): 2452-66.

7. Levine RL, Gilliland DG. Myeloproliferative disorders. Blood 2008; 112(6): 2190-8.

8. Barbui T, Barosi G, Grossi A, Gugliotta L, Liberato LN, Marchetti M, et al. Practice guidelines for the therapy of essential thrombocythemia. A statement from the Italian Society of Hematology, the Italian Society of Experimental Hematology and the Italian Group for Bone Marrow Transplantation. Haematologica 2004; 89(2): 215-32.

9. Cortelazzo S, Finazzi G, Ruggeri M, Vestri O, Galli M, Rodeghiero F, et al. Hydroxyurea for patients with essential thrombocythemia and a high risk of thrombosis. N Engl J Med 1995; 332(17): 1132-6.
10. Finazzi G, Barbui T. Efficacy and safety of hydroxyurea in patients with essential thrombocythemia. Pathol Biol 2001; 49(2): 167-9.

11. Landolfi R, Patrono C. Aspirin in polycythemia vera and essential thrombocythemia: current facts and perspectives. Leuk Lymphoma 1996; 22(Suppl 1): 83-6.

12. Simon HU, Rothenberg ME, Bochner BS, Weller PF, Wardlaw AJ, Wechsler ME, et al. Refining the definition of hypereosinophilic syndrome. J Allergy Clin Immunol 2010; 126: 45-9.

13. Tefferi A, Gotlib J, Pardanani A. Hypereosinophilic Syndrome and Clonal Eosinophilia:Point of Care Diagnostic Algorithm and Treatment Update. Mayo Clin Proc 2010; 85(2): 158-64.

14. Hoffman R, Wasserman LR. Natural history and management of polycythemia vera. Adv Intern Med 1979; 24: 255-85.

15. Barbui T. The leukemia controversy in myeloproliferative disorders: is it a natural progression of disease, a secondary sequela of therapy, or a combination of both? Semin Hematol 2004; 41(2 Suppl 3): 15-7.

16. Kiladjian JJ, Chomienne C, Fenaux P. Interferon-alpha therapy in bcr-ablnegative myeloproliferative neoplasms. Leukemia 2008; 22(11): 1990-8.

17. Landolfi R, Marchioli R. European Collaboration on Low-dose Aspirin in Polycythemia Vera (ECLAP): a randomized trial. Semin Thromb Hemost 1997; 23(5): 473-8.

18. Adir Y, Humbert M. Pulmonary hypertension in patients with chronic myeloproliferative disorders. Eur Respir J 2010; 35: 1396-406.

19. Holle N, de Witte T, Mandingers C, Schaap N, Raymakers R. Thalidomide and lenalidomide in primary myelofibrosis. Neth J Med 2010; 68: 7-8.

20. Huang JY, Tefferi A. Erythropoiesis stimulating agents have limited therapeutic activity in transfusion-dependent patients with primary myelofibrosis regardless of serum erythropoietin level. Eur J Haematol 2008; 83: 154-5.

21. Papadakis E, Hoffman R, Brenner B. Thrombohemorrhagic complications of myeloproliferative disorders. 2010; 24(6): 227-32

22. Landolfi R, Rocca B, Patrono C. Bleeding and thrombosis in myeloproliferative disorders: mechanisms and treatment. Crit Rev Oncol Hematol 1995; 20(3): 203-22.

23. Passamonti F. How I treat Polycythemia Vera. Blood 2012; 2: 366054

24. Verstovsek S, Mesa RA, Gotlib J, et al. A double-blind, placebo-controlled trial of ruxolitinib for myelofibrosis. N Engl J Med 2012; 366(9): 799-807 